\title{
Role of Computerized Tomography in Evaluation of Coronary Bypass Grafts-Special Reference to Women
}

\author{
Sujata Patnaik ${ }^{1}$ Sri Rama Murty ${ }^{1} \quad$ Amaresh Rao ${ }^{2}$ \\ ${ }^{1}$ Department of Radiology and Imageology, Nizam's Institute of \\ Medical Sciences, Punjaguta, Hyderabad, India \\ 2Department of CT Surgery, Nizam's Institute of Medical Sciences, \\ Punjaguta, Hyderabad, India
}

\author{
Susarla Rammurti ${ }^{1}$
}

Ind J Car Dis Wom 2020;5:57-64

\author{
Address for correspondence Sujata Patnaik, MD, Department \\ of Radiology and Imageology, Nizam's Institute of Medical \\ Sciences,Punjaguta,Hyderabad 500082, India \\ (e-mail: sujata_patnaik222@yahoo.co.in).
}

\begin{abstract}
Keywords

- CT-coronary angiography

- coronary-bypass graft

- arterial graft

- venous graft

- graft failure

Computerized tomography-coronary angiography (CT-CAG) is gaining popularity as an alternative to conventional CAG to evaluate grafts in a post-coronary artery bypass graft (post-CABG) patient, since it is a noninvasive procedure and is less influenced by cardiac motion. The primary challenge is to image a rapidly beating heart. With introduction of 64-slice scanner, the coronary imaging became a possibility with acceptable accuracy. In recent years, with the development of 128,256 and 320 multislice CT scanners, further enhancement in the temporal and spatial resolution is achieved due to lesser influence of the respiratory and cardiac motion, enhancing the accuracy of the lesion assessment in the grafts and the native vessels. Achieving low heart rate and artifact-free image acquisition, proper reconstruction, and image interpretation are challenges to the radiologists and the technicians involved in coronary imaging. Women pose special subset because of smaller sized coronary vessels, interference due to breast shadows, low-referrals, and gender-specific reluctance to accept the procedure itself. Cardiologists and radiologists caring for these patients must be familiar with the pros and cons of CT-CAG and gender-specific challenges.
\end{abstract}

\section{Introduction}

Computerized tomography-coronary angiography (CT-CAG) is an emerging imaging tool to rule out significant stenosis in suspected cases of coronary artery disease (CAD). Conventional CAG for graft assessment is considered the gold-standard; however, it is not acceptable to most of the patients due to its invasive nature, more so because the femoral route has to be used. Though rare, the conventional coronary angiograms have potential complications like stroke, dissection, infarction, and arrhythmia. CT-CAG is gaining popularity as an alternative, since it is a noninvasive procedure and is less influenced by cardiac motion especially due to introduction of new generation of CT scanners. CT-CAG is mainly indicated for diagnosis of intermediate-low probability CAD and to evaluate in-stent or coronary-bypass graft disease or when vasculitis or aneurysm is suspected. The need for assessment of the coronary bypass grafts arises when there is recurrence of angina or when inducible ischemia is demonstrated.

\section{Types of Grafts}

Based on the source of the conduits, the grafts are either arterial or venous. Great saphenous vein is the most commonly used vessel for the grafts. Harvested saphenous veins are used as venous conduits from ascending aorta (usually anterior aspect) to the coronary artery beyond the obstructive lesion (right coronary artery [RCA], posterior descending coronary artery [PDA], left anterior descending artery [LAD], left circumflex coronary artery [LCX], and obtuse marginal $[\mathrm{OM}]$ ). Generally, an accepted sequence [right side from above downward as saphenous vein graft (SVG) to RCA/PDA and on left they are LCX, OM, LAD from above downward] is followed 
by most surgeons to avoid cross over and kinking. ${ }^{1}$ Though it is technically easier to perform SVG, they are more prone to degeneration and occlusion. As venous grafts are less pulsatile, wider in diameter (approximately $4-10 \mathrm{~mm}$ vs. $2-5 \mathrm{~mm}$ of the native arteries), and less prone to calcification, they are better suited for study using CT-CAG. Arterial grafts are taken from left or right internal mammary arteries (LIMA or RIMA), free radial artery, inferior epigastric, or gastroepiploic arteries. Depending on the technique of placing them they are described as sequential grafts, jump-grafts, y-grafts, or T-grafts (-Figs. 1-4). Arterial grafts have better adaptability to high systemic pressure and shear stress. The patency rate of arterial grafts is approximately $96.5 \%$ at 15 years whereas venous grafts have an attrition rate of 2 to $5 \%$ per year. ${ }^{2,3}$ The 10 -year patency is as high as $88 \%$. The patency of arterial grafts also depends on the status of native vessels. If size of native vessel is more than $2 \mathrm{~mm}$ and the stenosis is more than $50 \%$ the results will be favorable. ${ }^{4}$

\section{Key Issues in Coronary Imaging}

The primary challenge is to image a rapidly beating heart. Hence the scanner should have high temporal resolution. That is made possible by introduction of newer generation CT scanners in the recent years. The 64-slice CT scanner has temporal resolution up to 83 milliseconds and spatial resolution up to $0.4 \times 0.4 \times 0.4 \mathrm{~mm}^{3}$. ${ }^{5}$ In recent years, with the introduction of 128,256 and 320 multislice CT further improvement in the temporal and spatial resolution is achieved with lesser respiratory and cardiac motion, which greatly enhanced the precision of the lesion assessment in the grafts and the native vessels. ${ }^{6,7}$

The most quiescent part of cardiac cycle is the diastolic phase and image acquisition is best performed in this phase. As heart rate increases the diastolic phase reduces. At heart rates below 65 beats/min imaging can be best performed. Diameter of coronary segments varies from millimeter to submillimeter. Hence, scanner should have the thinnest slice thickness to obtain isotropic images. In other words, the scanners with 64 slices onward are best suitable for coronary imaging. Overall, CT-CAG using 64 slices CT scanner was proven to have superior sensitivity and specificity compared with invasive conventional CAG in coronary bypass graft stenosis or occlusion evaluation. Earlier venous graft disease detection and evaluation of extracardiac anatomical features are additional advantages of CT-CAG. ${ }^{8}$

\section{Limitations of CT-CAG}

Gross obesity and irregular cardiac rhythm are important limitations to CT-CAG. Patients with many metallic clips and

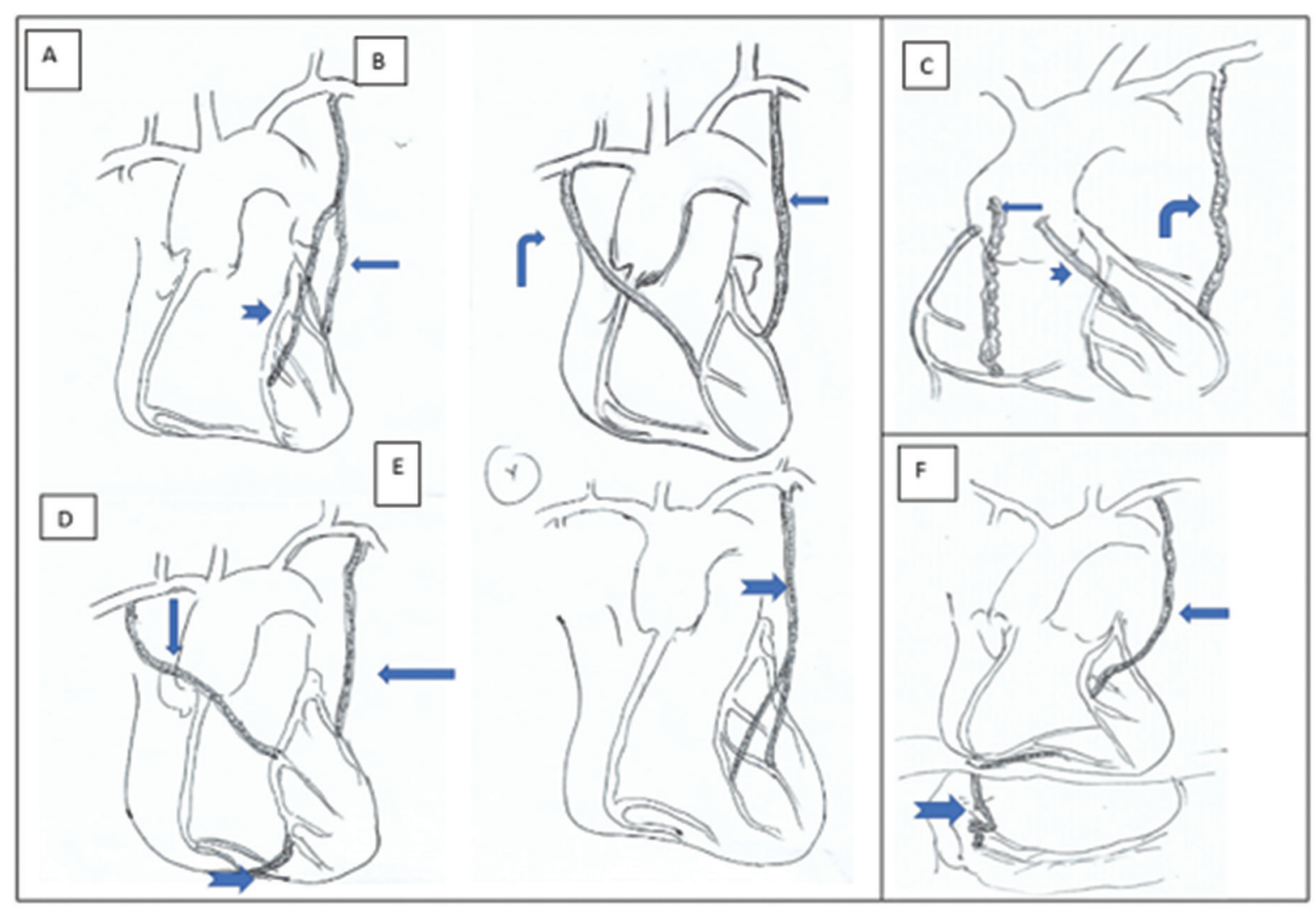

Fig. 1 Diagrammatic representation of various grafts: (A) LIMA to OM (thin arrow) and free radial artery graft from middle of LIMA to LAD (broad arrow); (B) RIMA to LAD (angled arrow) and LIMA to LCX (straight arrow); (C) Angled arrow showing LIMA to LAD, broad short arrow showing free radial graft to diagonal artery and SVG to distal RCA; (D) RIMA to LAD, jump graft from LAD to RCA (broad arrow), LIMA to LAD (thin arrow); (E) "Y" graft to distal LAD and D2 (broad arrow); (F) LIMA to LAD (thin arrow) and gastroepiploic artery to distal RCA (broad arrow). LAD, left anterior descending artery; LCx, left circumflex coronary artery; LIMA, left internal mammary arteries; OM, obtuse marginal; RCA, right coronary artery; RIMA, right internal mammary arteries; SVG, saphenous vein graft. 

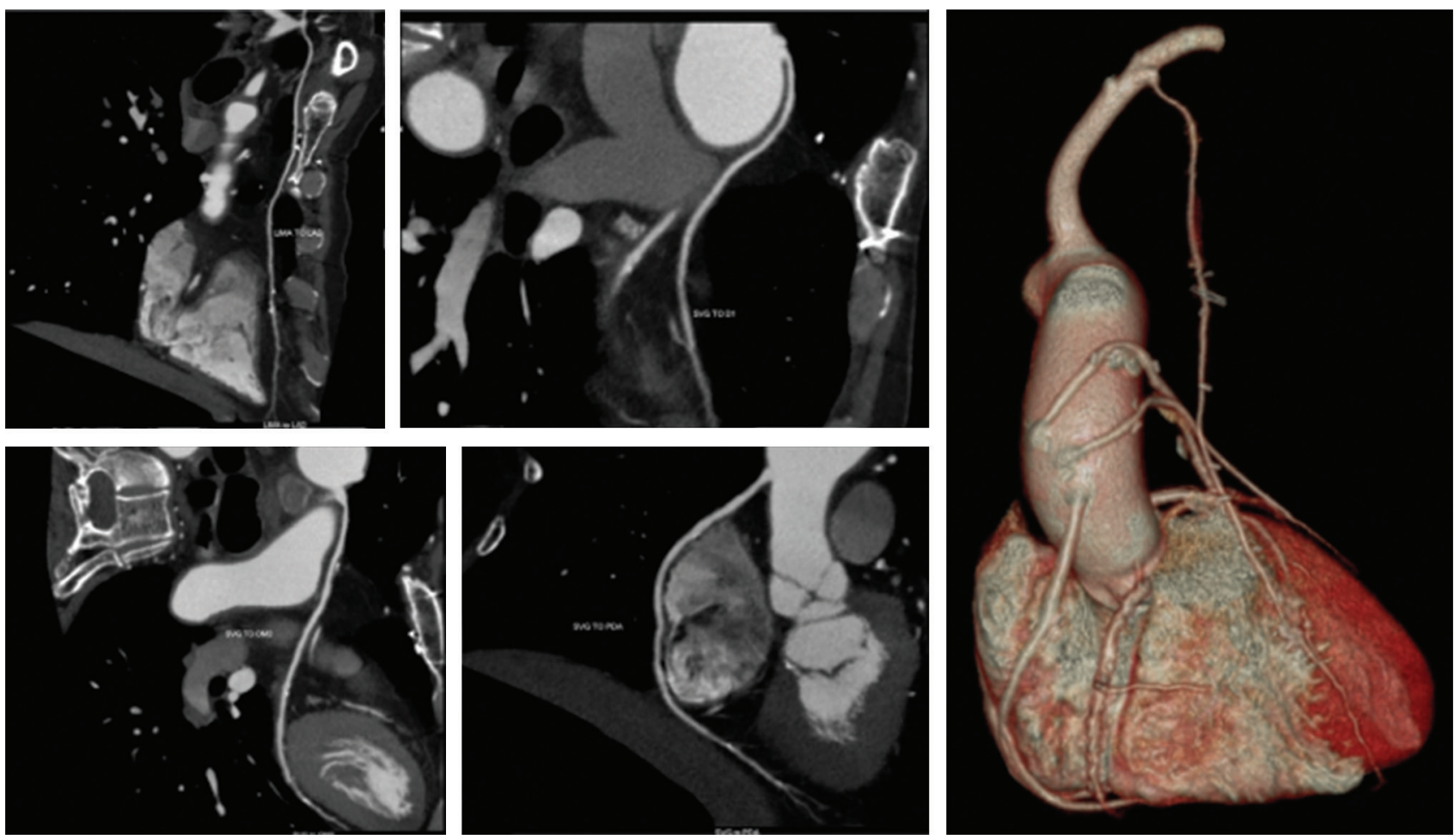

Fig. 2 CT-CAG showing LIMA to LAD, SVG to D1, OM2 and PDA. Observe the sequence of SVGs. CT-CAG, computerized tomography-coronary angiography; LAD, left anterior descending artery; LIMA, left internal mammary arteries; OM2, obtuse marginal 2; PDA, posterior descending coronary artery; SVG, saphenous vein graft.

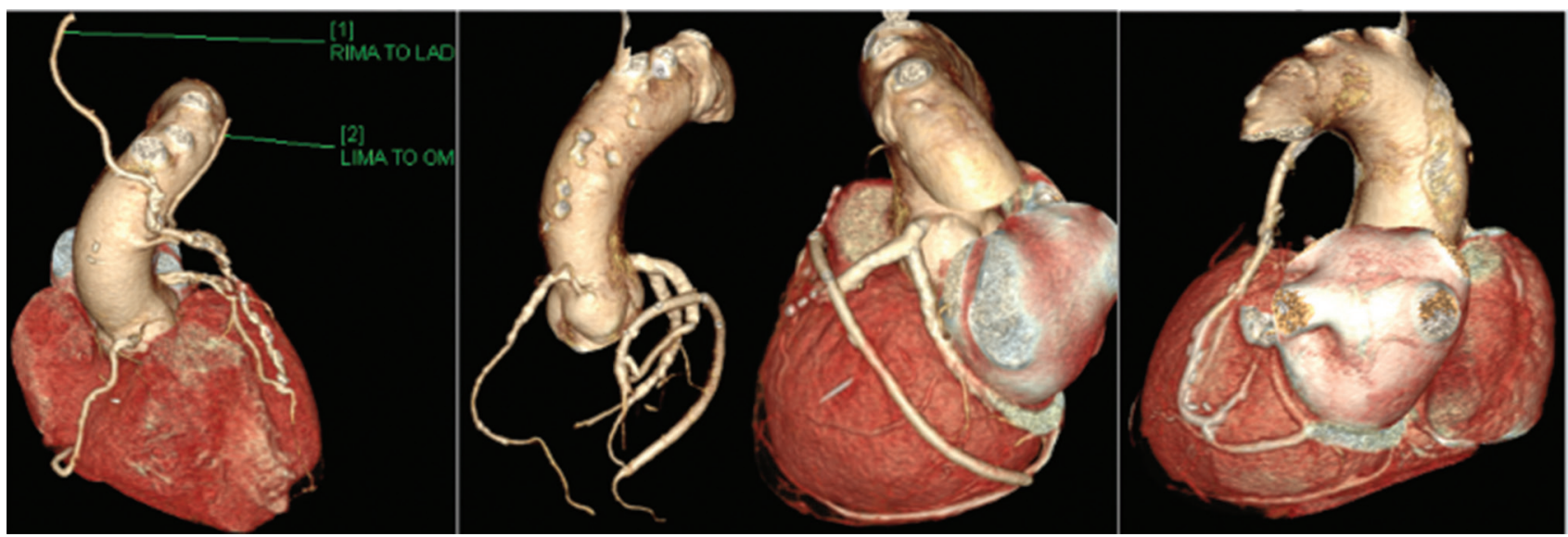

Fig. 3 CT-CAG showing RIMA to LAD, LIMA to OM1, Jump-graft from OM3 to LAD in patient (70, male) and sequential graft from SVG to PDA and LCX in a 50-year-old female. CT-CAG, computerized tomography-coronary angiography; LAD, left anterior descending artery; LCX, left circumflex coronary artery; LIMA, left internal mammary arteries; OM1, obtuse marginal 1; PDA, posterior descending coronary artery; RIMA, right internal mammary arteries; SVG, saphenous vein graft.

those with estimated glomerular filtration rate, $30 \mathrm{~mL} / \mathrm{min}$ are also challenging subsets. As the CT-CAG is a nondynamic study, competitive flow and vasospasm in arterial grafts may be difficult. Evaluation of native vessels and any flow-limiting stenosis in them may be more difficult on CT-CAG (compared with the grafts). ${ }^{9}$

\section{Technical Aspects}

The study of grafts by CT-CAG requires a larger scanning range (up to $22 \mathrm{~cm}$ ) and hence a longer breath holding. Recent scanners have faster gantry rotation, wide field of scan at a higher X-ray tube potential but at a lower patient radiation dose and better images. Scan range must be extended from supraclavicular fossa to base of heart/diaphragm so that the origin of internal mammary artery can be visualized. Breath holding is longer (approximately $15 \mathrm{~s}$ ) than usual chest imaging. Heart rate should be brought below 65 beats/min by use of $\beta$-blockers or ivabradine. The reconstructions like volume rendering technique (VRT), multiplanar reformations (MPRs), cMPR, and maximum intensity projection (MIP) have to be made for analysis. Special care has to be taken for patients with stents or heavy calcification of coronaries. History of pregnancy and contrast allergy is to be obtained 

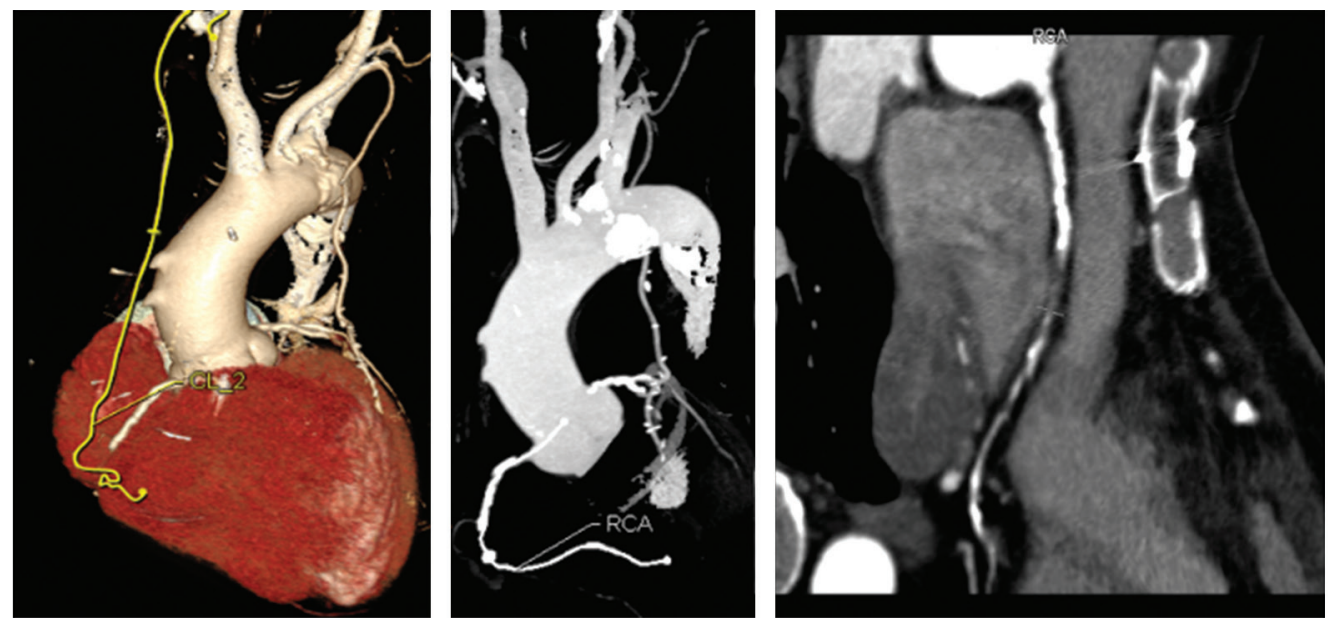

Fig. 4 CT-CAG showing patent LIMA to LAD; But SVG to RCA is occluded-it is identified by the surgical clips and the vessel calcification. CTCAG, computerized tomography-coronary angiography; LAD, left anterior descending artery; LIMA, left internal mammary arteries; RCA, right coronary artery; SVG, saphenous vein graft.

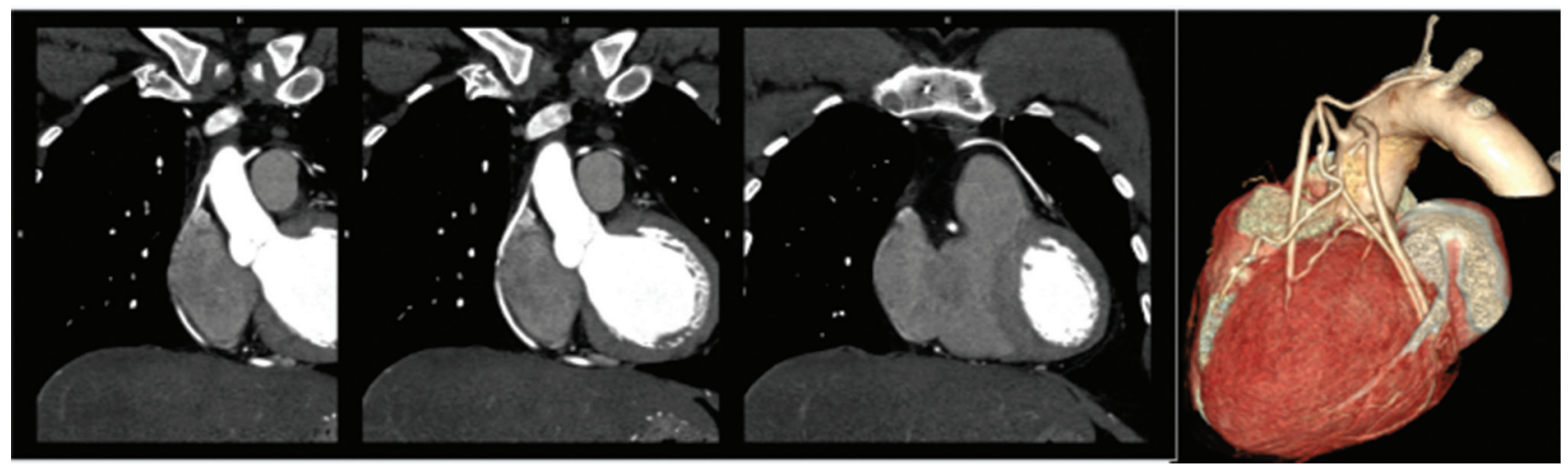

Fig. 5 CT-CAG showing patent LIMA to LAD and patent SVGs to D1, OM, PDA, continuous contrast column indicates patent graft. CT-CAG, computerized tomography-coronary angiography; LAD, left anterior descending artery; LIMA, left internal mammary arteries; OM, obtuse marginal; PDA, posterior descending coronary artery; SVG, saphenous vein graft.

before performing the scan. Medications like sildenafil and metformin have to be stopped on the day of examination. Four-hour fasting is to be ensured but dehydration has to be avoided. Patient should abstain from caffeine $12 \mathrm{~h}$ before on the day of examination. Just before starting the scan nitroglycerine can be administered to dilate coronaries. IV contrast is injected by bolus tracking. The recommended dose (64 slice CT with dual head injector) is contrast agent containing 270 to $400 \mathrm{mg}$ iodine $/ \mathrm{mL}$ at the rate of 5 to $7 \mathrm{~mL}$ contrast/s; total up to $120 \mathrm{~mL}$ was administered followed by saline chaser. Radiation dose is approximately 8 to $22 \mathrm{mSV}$ in retrospective echocardiographic gating and much lesser in prospective gating. ${ }^{10}$

\section{Imaging Interpretation}

Axial, sagittal, coronal views are to be critically viewed. Systemic approach was to be adopted to evaluate the chest wall for metallic clips, sternal dehiscence, and osteomyelitis. Comprehensive graft evaluation along with the native coronaries has to be performed by three-dimensional VRT and all other reconstructions like MPRs and curved-MPR. The stenoses are assessed by scrolling through the axial images with or without MIP. When there is homogenous enhancement with smooth walls the graft is said to be patent. It may not be possible to see the distal anastomosis in all situations; however, continuous contrast column in the graft can be taken to indicate a patent graft. Distal native vessel-runoff is also to be assessed in all cases (-Figs. 4-6). All radiologists involved in CT-CAG should be aware of certain artifacts that are occasionally encountered due to patient, procedure, and reconstruction-related factors. By appropriate patient counseling, modification of technique, and machine parameters these can be reduced or avoided. ${ }^{11,12}$

\section{Graft Failure}

Stenosis or occlusion is assessed by calculating diameter stenosis. Plaques (calcified, noncalcified plaques, mixed plaques) can also be assessed. Nonvisualization of graft indicates occlusion. Ghost or Nubbin sign is small contrast filled out pouch at the graft site ( - Fig. 4 ). It indicates total occlusion of the proximal site. Graft failure is said to present if there is narrowing more than $75 \%$ or total occlusion 

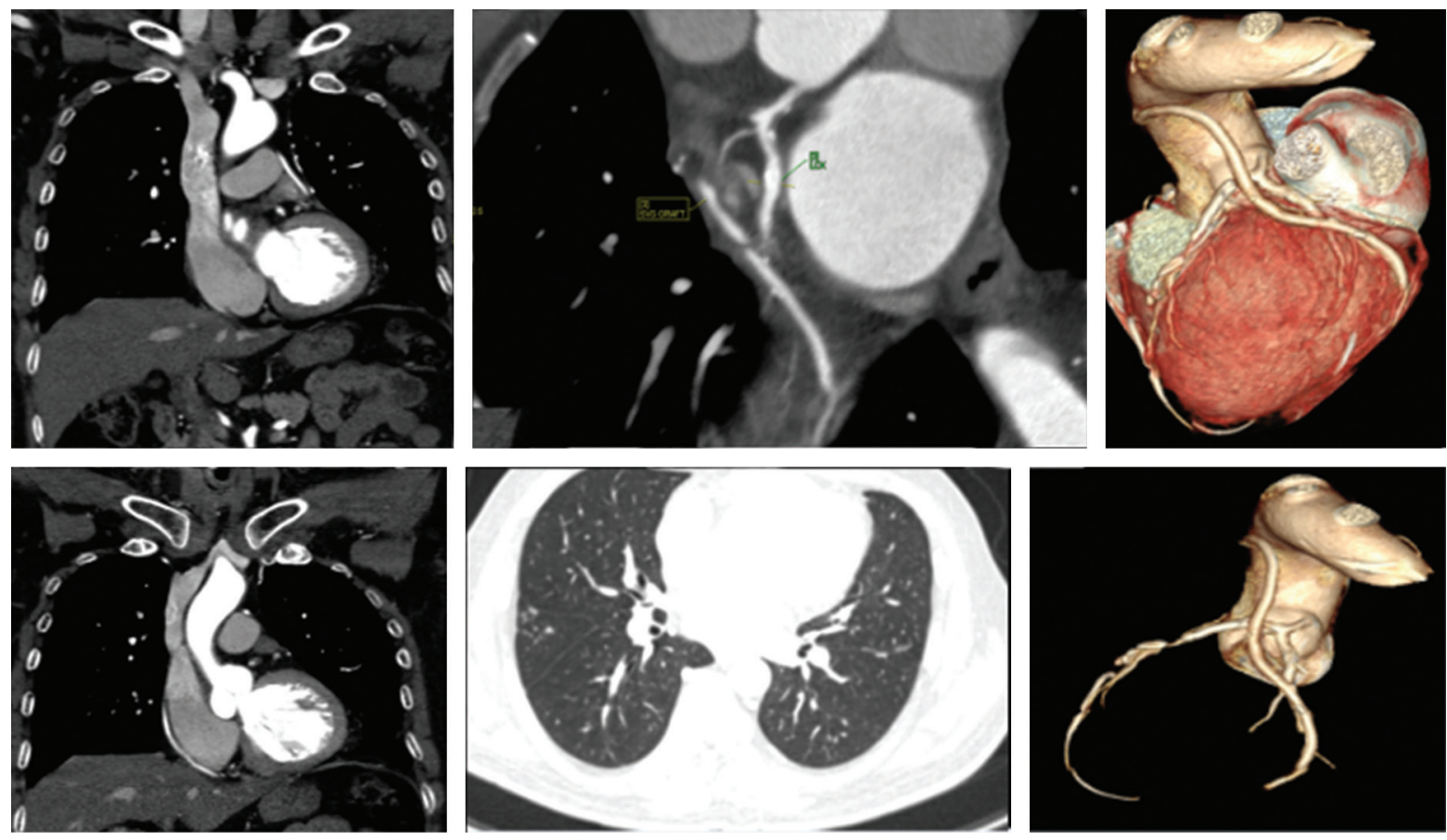

Fig. 6 CT-CAG showing patent LIMA to LAD, SVG to OM, and PDA; also, can see lung nodules bilaterally. CT-CAG, computerized tomography-coronary angiography; LAD, left anterior descending artery; LIMA, left internal mammary arteries; OM, obtuse marginal; PDA, posterior descending coronary artery; SVG, saphenous vein graft.

and not visualized. Graft failure in first few weeks is due to either technical errors or due to thrombus. Graft malfunction can also manifest in the form of twist, kink, stretching, or shortening. Graft spasm may mimic narrowing. Free radial artery grafts are more prone to spasm. Spasm occurs more common in early postoperative period (within 1 month). Here the proximal segment is narrowed as compared with distal segment whereas in graft occlusion the distal segment appears narrower than proximal segment. Within 1-year, intimal hyperplasia and thrombosis are common causes of occlusion. After 1 year, the neointimal atherosclerosis is the predominant cause of graft failure, whereas after 5 years degeneration and chronic thrombosis account for 40 to $60 \%$ graft failure. ${ }^{13-15}$

\section{Other Postsurgical Complications}

CT-scan can detect several noncoronary complications that can occur in a post-CABG patient. Pericardial effusion can occur in more than $75 \%$ cases and anticoagulants are common offenders. It occurs in first 5 to 10 days of postoperative stage and resolves within few weeks. Only $0.3 \%$ develops tamponade. Pleural effusion occurs in $89 \%$ cases and usually left-sided and minimal. Sternal dehiscence and or infection occurs in 0.3 to $0.5 \%$ ( - Fig. 7 ). When lucency between the incision site is more than $3 \mathrm{~mm}$, it indicates dehiscence. Osteomyelitis may show irregularity of bone with adjacent soft tissue. Wire malposition, fractures and pseudoarthrosis, mediastinitis, and pseudoaneurysm are other uncommon complications. Very rarely there may be true or pseudoaneurysmal formation at the grafted

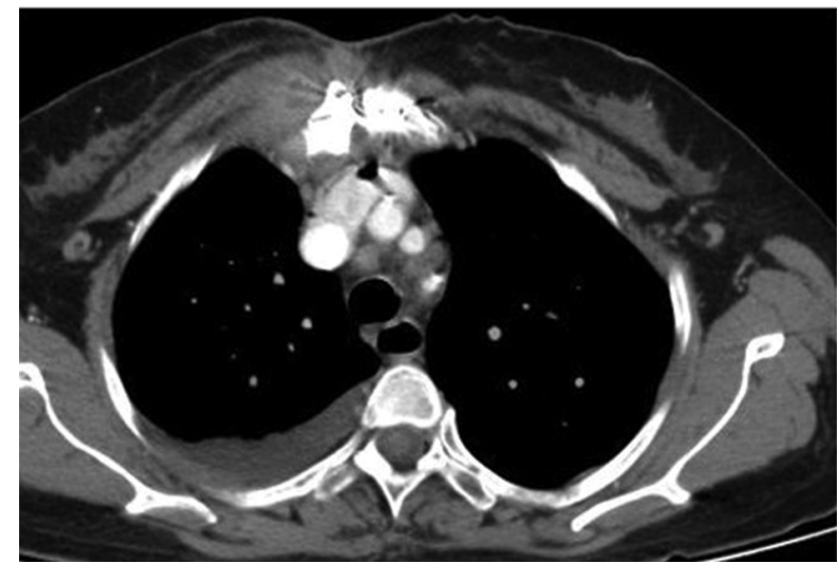

Fig. 7 Sternal osteomyelitis and pleural effusion in a patient who underwent CT-CAG for graft evaluation. CT-CAG, computerized tomography-coronary angiography.

site. Pseudoaneurysms follow local infection or tension in early postoperative period. True aneurysms may present later due to atherosclerosis and rarely complicate. Other common findings that are observed incidentally are pulmonary thromboembolism, pulmonary nodules, atelectasis, and pneumothorax ( - Figs. 6 and 7 ).6,16,17

\section{Role in Special Situations}

For planning for re-do-CABG, CT-CAG has an additional value in providing the mediastinal anatomy. The number, position, the course of grafts as well as distance of LIMA to sternum are important points to be assessed before redo surgery and are 


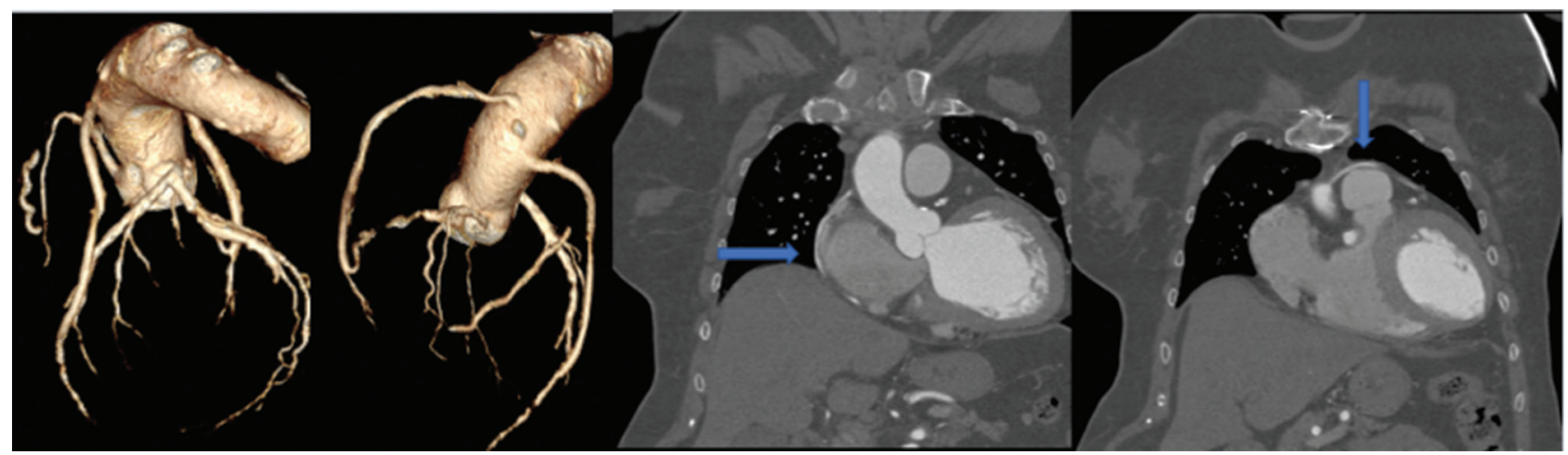

Fig. 8 CT-CAG showing patent SVG to LAD, and PDA in a female patient. CT-CAG, computerized tomography-coronary angiography; LAD, left anterior descending artery; PDA, posterior descending coronary artery.

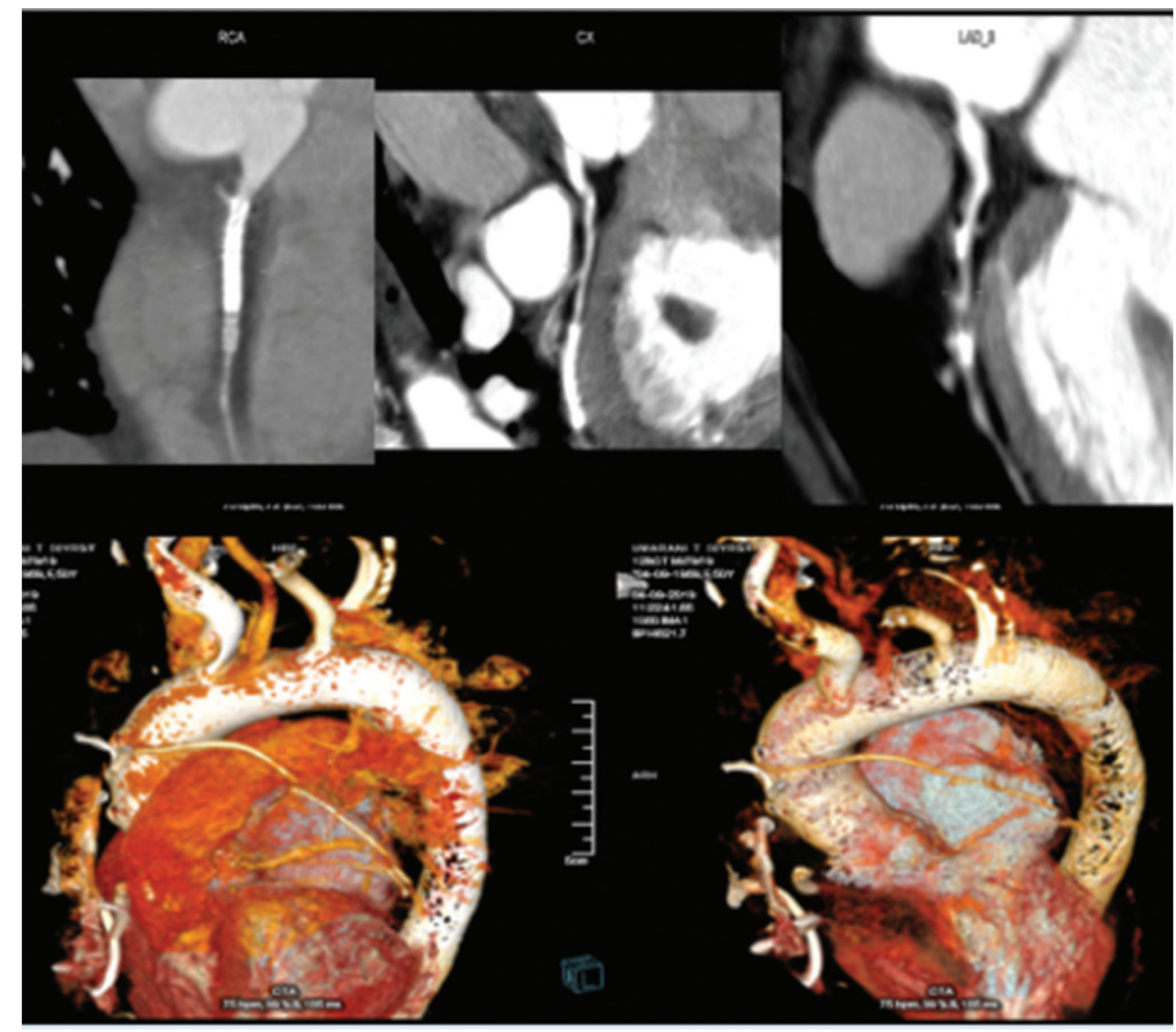

Fig. 9 CT-CAG showing native vessels with stents patent SVG to LAD and occluded SVGs to RCA and LCX in a female patient. CT-CAG, computerized tomography-coronary angiography; LAD, left anterior descending artery; LCx, left circumflex coronary artery; RCA, right coronary artery; SVG, saphenous vein graft.

best evaluated using the CT-CAG compared with the conventional CAG. It is also an invaluable tool for minimally invasive CABG, off-pump CABG, and for evaluating before all arterial bypass grafts. ${ }^{18}$

\section{Our Experience in NIMS}

At NIMS (Nizam's Institute Of Medical Sciences), we had performed 52 CT-CAGs since January, 2018. All our cases come for evaluation from Cardiology/Cardiothoracic and Vascular Surgery departments when the doctor treating the case feels it is needed. Surprisingly, of the 52 studied cases there were only 8 females (15.4\%). The mean ages of the whole group, males and females were 62.9, 63.1, and 61.5 years, respectively.

\section{Women-Specific Issues}

CAD is the number one killer both in women and men. There are a few gender-specific differences in the presentation and prognosis of this disease. Females are supposed to have relative protection from obstructive CAD in their 


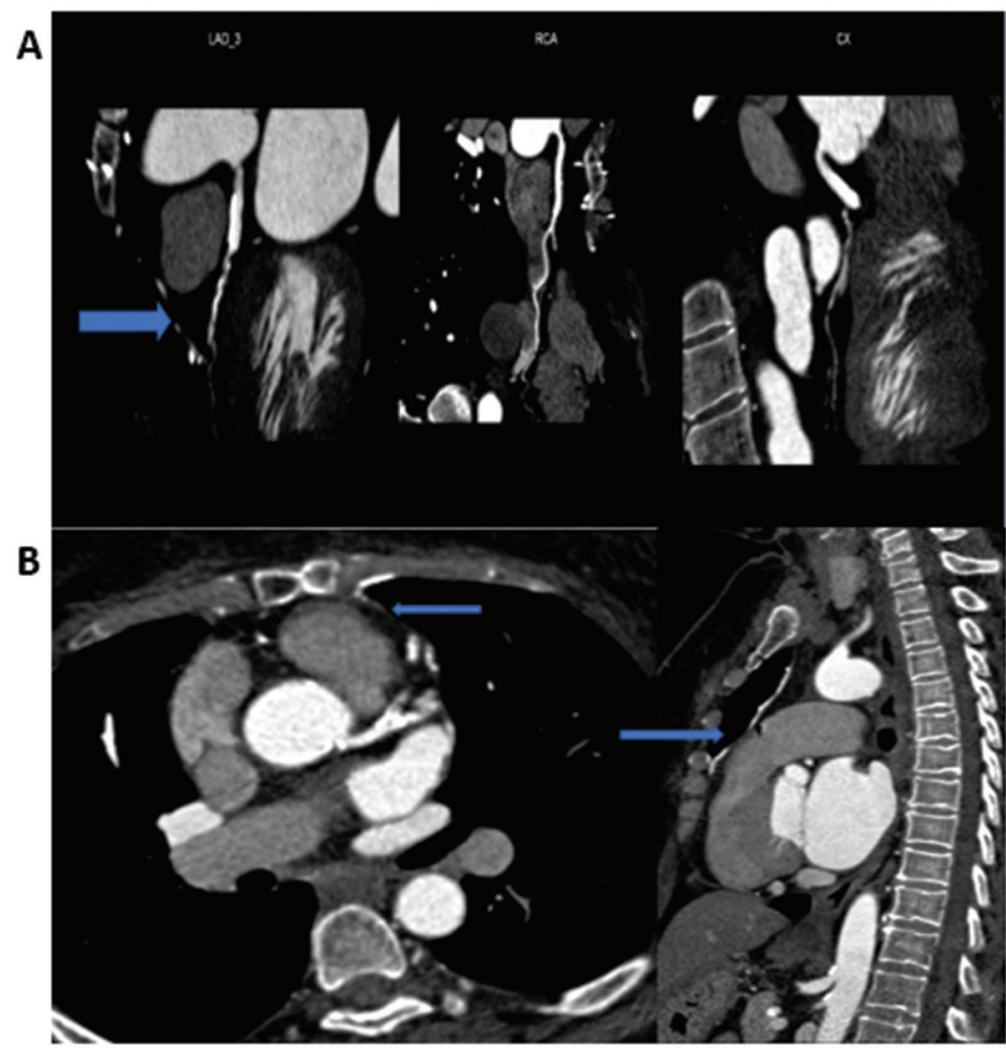

Fig. 10 (A) CT-CAG showing occluded LIMA to LAD in a 66-year-old female; (B) CT-CAG showing patent LIMA to LAD in a female patient. CTCAG, computerized tomography-coronary angiography; LAD, left anterior descending artery; LIMA, left internal mammary arteries.

premenopausal phase but when affected, the angina and the mortality due to CAD is higher in them. The National Heart, Lung, and Blood Institute (NHLBI) data suggest that 1-year mortality after myocardial infarction is $19 \%$ in men versus $26 \%$ in women and after 5 years the respective figures are 36 and $47 \%$, respectively. ${ }^{19}$ This paradox is attributed to a different path physiology, nonstandardized risk-estimation tools, difference in performing the evaluation, and acceptance of surgery as an option, and difficulties in imaging due to higher prevalence of obesity and breast shadow (-Fig. 8). ${ }^{20}$ Women have smaller sized coronary arteries (-Fig. 9), smaller left ventricular chamber dimensions, and greater chest wall attenuation due to breast tissue. These factors may increase the chance of nonevaluability of images obtained with coronary CT angiography. ${ }^{21}$ However, in an elaborate study coronary angiography in men and women had a sensitivity of 90 and $85 \%$ and a specificity of 89 and $78 \%$, respectively which are quite comparable ( - Fig. 10). ${ }^{22}$ The number of women included at our center is surprisingly far below the expectation. It highlights the fact that underreporting of symptoms and underutilization of expensive investigations and therapies are quite common among our women. There is also a gender-bias in physician referral for CT-CAG.

\section{Conclusion}

Women with prior CABG comprise a very special population with unique imaging and diagnostic considerations.
CT-angiography is recently proven as a competitive alternative to conventional CAG for bypass graft evaluation. Cardiologists and radiologists taking care of these patients must be familiar with the pros and cons and some basic facts about CT-CAG. They should also realize gender-specific challenges to use this wonderful diagnostic tool more widely and appropriately in the women after CABG.

\section{Conflict of Interest}

None declared.

\section{References}

1 Parsa CJ, Daneshmand MA, Gaca JG, et al. Arterial bypass grafting of the coronary circulation. HSR Proc Intensive Care Cardiovasc Anaesth 2011;3:227:234

2 Tatoulis J, Buxton BF, Fuller JA. Patencies of 2127 arterial to coronary conduits over 15 years. Ann Thorac Surg 2004;77(1):93-101

3 Sabik JF II. Understanding saphenous vein graft patency. Circulation 2011;124(3):273-275

4 Owens CD. Adaptive changes in autogenous vein grafts for arterial reconstruction: clinical implications. J Vasc Surg 2010;51(3):736-746

5 Nazeri I, Shahabi P, Tehrai M, Sharif-Kashani B, Nazeri A. Assessment of patients after coronary artery bypass grafting using 64-slice computed tomography. Am J Cardiol 2009;103(5):667-673

6 Dewey M, Zimmermann E, Deissenrieder F, et al. Non-invasive coronary angiography by 320 row CT with lower radiation exposure and maintained diagnostic accuracy. Circulation 2009;120:867-875 
7 Chao SP, Leu JG, Law WY, Kuo CJ, Shyu KG. Image quality of 256-slice computed tomography for coronary angiography. Acta Cardiol Sin 2013;29(5):444-450

8 Barbero U, Iannaccone M, d'Ascenzo F, et al. 64 slice-coronary computed tomography sensitivity and specificity in the evaluation of coronary artery bypass graft stenosis: a meta-analysis. Int J Cardiol 2016;216:52-57

9 Dewey M, Cardiac CT. 2nd ed. Berlin Heidelberg: Springer; 2014

10 Donkol RH, Mahmoud ZS, MohamedE. Evaluation of coronary artery bypass by CT coronary angiography. In: Coronary Artery Bypass Graft Surgery; 2017:1-23 Available at: http://dx.doi. org/10.5772/intechopen.70439. Accessed December 26, 2019

11 Telkar HB. Pictorial essay: artefacts in cardiac CT: a practical system of understanding and correction. Indian J Radiol Imaging 2007;17:29-36 [serial online]

12 Sundaram B, Quint LE, Patel HJ, Deeb GM. CT findings following thoracic aortic surgery. Radiographics 2007;27(6):1583-1594

13 Patra S, Jena M, Pande A, Ghosh D, Chakraborty RN. Assessment of coronary artery bypass grafts status in symptomatic patients: an observational study. J Cardiovasc Thoracic Surg 2017;2(1):4

14 Hemat EM, Zidan EH, Ismail AA, Khalil WA. Multislice CT angiography assessment of coronary artery bypass graft (CABG) patients. J Am Sci 2016;12(8):92-98

15 Cao C, Ang SC, Wolak K, Peeceeyen S, Bannon P, Yan TDA. A meta-analysis of randomized controlled trials on mid-term angiographic outcomes for radial artery versus saphenous vein in coronary artery bypass graft surgery. Ann Cardiothorac Surg 2013;2(4):401-407
16 Bitkover CY, Cederlund K, Aberg B, Vaage J. Computed tomography of the sternum and mediastinum after median sternotomy. Ann Thorac Surg 1999;68(3):858-863

17 Pesenti-Rossi D, Baron N, Georges JL, Augusto S, Gibault-Genty G, Livarek B. Assessment of coronary bypass graft patency by first-line multi-detector computed tomography. Ann Cardiol Angeiol (Paris) 2014;63(5):284-292

18 Flemy LM, Multidetector CT Angiography for coronary Bypass Graft Assessment and Reoperative Cardiac Surgery. CT of the Heart (Contemporary Medical Imaging Book Series); 2019 381-390

19 Go AS, Mozaffarian D, Roger VL, et al; American Heart Association Statistics Committee and Stroke Statistics Subcommittee. Heart disease and stroke statistics-2014 update: a report from the American Heart Association. Circulation 2014;129(3):e28-e292

20 Bairey Merz CN. Women and ischemic heart disease paradox and pathophysiology. JACC Cardiovasc Imaging 2011;4(1):74-77

21 Nevsky G, Jacobs JE, Lim RP, Donnino R, Babb JS, Srichai MB. Sex-specific normalized reference values of heart and great vessel dimensions in cardiac CT angiography. AJR Am J Roentgenol 2011;196(4):788-794

22 Tsang JC, Min JK, Lin FY, Shaw LJ, Budoff MJ. Sex comparison of diagnostic accuracy of 64-multidetector row coronary computed tomographic angiography: results from the multicenter ACCURACY trial. J Cardiovasc Comput Tomogr 2012;6(4):246-251 